\title{
Fatal Interstitial Lung Disease Associated With Erlotinib: A Case Report and Review of Literature
}

\author{
Ling-chuan Guo ${ }^{1}$, Xi-ming Wang ${ }^{2}$, Sheng-ming Deng ${ }^{3}$, Yan-bin Chen ${ }^{4}$ \\ ${ }^{1}$ Department of Pathology, the First Affiliated Hospital of Soochow University, Suzhou, 215006, China \\ ${ }^{2}$ Department of Radiology, the First Affiliated Hospital of Soochow University, Suzhou, 215006, China \\ ${ }^{3}$ Department of Nuclear Medicine, the First Affiliated Hospital of Soochow University, Suzhou, 215006, China \\ ${ }^{4}$ Department of Respiratory Medicine, the First Affiliated Hospital of Soochow University, Suzhou, 215006, China
}

${ }^{*}$ Corresponding author: Yan-bin Chen, Department of Respiratory Medicine, the First Affiliated Hospital of Soochow University, 188\#, Shizi street, Suzhou, 215006, China. Tel: 0086 13814881257; Fax: 0086512 65225636; Email: chen001chen@163.com

Received Date: February 27, 2015; Accepted Date: April 22, 2015; Published Date: April 24, 2015

Citation: Ling-chuan Guo, et al. (2015) Fatal Interstitial Lung Disease Associated With Erlotinib: A Case Report and Review of Literature. J Cancer Res Therap Oncol 1: 1-4

\begin{abstract}
Erlotinib is a human epidermal growth factor receptor / tyrosine kinase inhibitor (EGFR-TKI), which is effective as a first and second-line treatment for patients with advanced stage non-small cell lung cancer (NSCLC) with EGFR mutations. Erlotinib is considered to have a favorable safety profile, few cases of interstitial lung disease (ILD) related to erlotinib have been described. We report a case of a 58-year-old male with stage IV NSCLC treated with erlotinib who developed ILD and died on $55^{\text {th }}$ day since commencing erlotinib. Respiratory symptoms during treatment with erlotinib should alert clinicians to rule out pulmonary toxicity, early erlotinib withdrawal is crucial.
\end{abstract}

Keywords: Drug-induced lung injury, Interstitial lung disease, Epidermal growth factor receptor tyrosine kinase inhibitor, Erlotinib, Non-small cell lung cancer

\section{Introduction}

Erlotinib is a reversible EGFR-TKI, which has been available for the treatment of patients with NSCLC since 2004 and has been included in NCCN guideline since 2010 as first-line treatment option for advanced NSCLC patients who harbor EGFR mutation [1]. From then on, erlotinib has opened up a new era of effective and relatively safe treatment of patients with advanced NSCLC $[2,3]$. Recently, the first case of erlotinib-induced ILD ( eILD), diagnosed based on clinical and radiologic findings, was reported [4]. Herein, we report a case of a NSCLC patient treated with erlotinib who developed ILD and died.

\section{Case Report}

A 58-year-old male patient with complaint of sacrococcygeal pain for about 3 months was admitted to our hospital in $20^{\text {th }}$ December, 2010. He had a history of cigarette smoking (1 pack/day for 35 years). Physical examination revealed normal vital signs except percussion pain in $2^{\text {nd }}$ sacral vertebrae

(C2015 The Authors. Published by the JScholar under the terms of the Creative Commons Attribution License http://creativecommons.org/licenses/by/3.0/, which permits unrestricted use, provided the original author and source are credited.
(S2) level. Pelvic computed tomographic (CT) scan showed lytic lesion in the right side of S2 (Figure 1A). Chest CT scan found a nodule with lobulated and speculated margin in the superior segment of the left lower lobe (Figure 1B,1C). S2 punctured biopsy followed by excision of sacral neoplasms (Figure 1D-1G, Figure 2A,2B) revealed a diagnosis of metastasis non-small cell cancer, derived from lung. So the definite diagnosis was lung adenocarcinoma (T2aNxM1b; stage IV) without EGFR mutation or chromosomal rearrangements of anaplastic lymphoma kinase (ALK) . He received cisplatin $\left(75 \mathrm{mg} / \mathrm{m}^{2}\right)$ and pemetrexed $\left(500 \mathrm{mg} / \mathrm{m}^{2}\right)$ every 3 weeks as an initial anticancer treatment. After four cycles of chemotherapy, CT scan showed a stable disease condition (Figure 2C,2D), so 4 cycles of maintenance therapy of pemetrexed $\left(500 \mathrm{mg} / \mathrm{m}^{2}\right)$ every 3 weeks were given subsequently. But positron emission tomography-computed tomography (PET-CT) detection revealed extensive metastasis in the left pleura (Figure 3A,3B,3C) after the above therapy, and disease progression was documented. The patient was not eligible for any other chemotherapy due to his poor performance status (PS), and the treatment of choice remained EGFR-TKI (erlotinib, $150 \mathrm{mg}$ once daily) in spite of his EGFR gene was wild, along with pleural fluid drainage. High-resolution CT (Figure 3D,3E) showed ground-glass opacities in both lungs, 


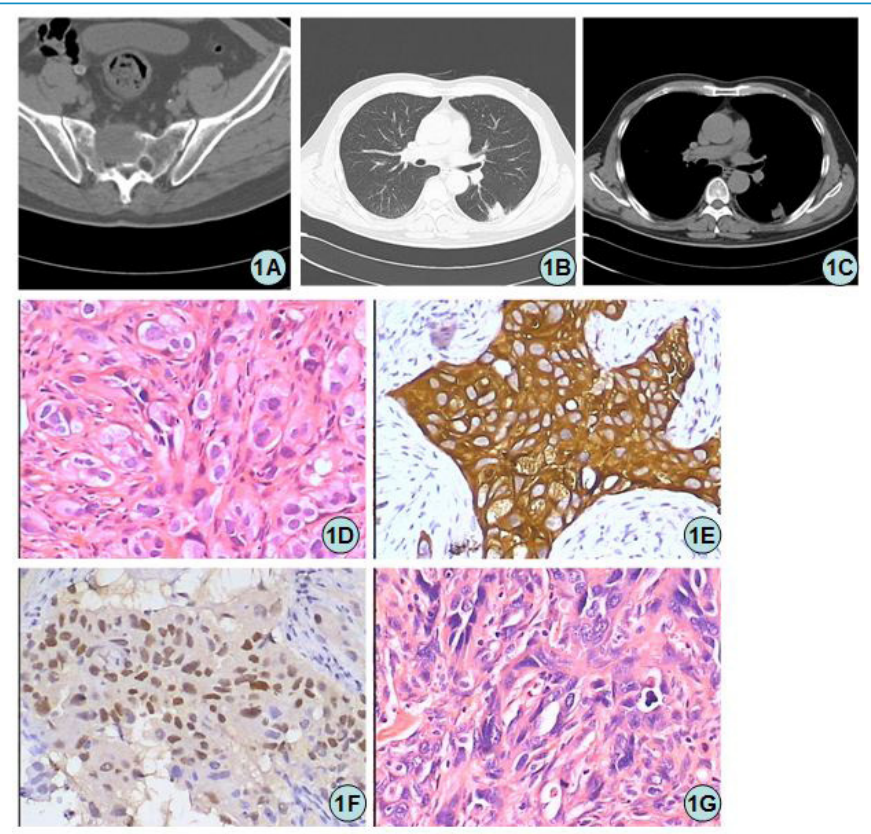

Figure 1:

Figure 1A: CT scan at the S2 level shows a lytic soft-tissue mass within the right side of the sacrum with erosion of the anterior cortex and extending into the right anterior sacral foramen. Figure 1B,1C: CT scan (lung window) obtained at the level of the left main bronchus reveals a nodule with lobulated and speculated margin in the superior segment of the left lower lobe. The nodule also can be seen in the mediastinal window. Figure 1D-1E: 1D HE staining of S2 punctured biopsy specimen in accordance with adenocarcinoma $(\times 40)$; $1 \mathrm{E}$ CK7 positive $(\times 20)$; $1 \mathrm{~F}$ TTF- 1 positive $(\times 20)$; $1 \mathrm{G} \mathrm{HE}$ staining of surgery biopsy specimen in accordance with adenocarcinoma $(\times 40)$
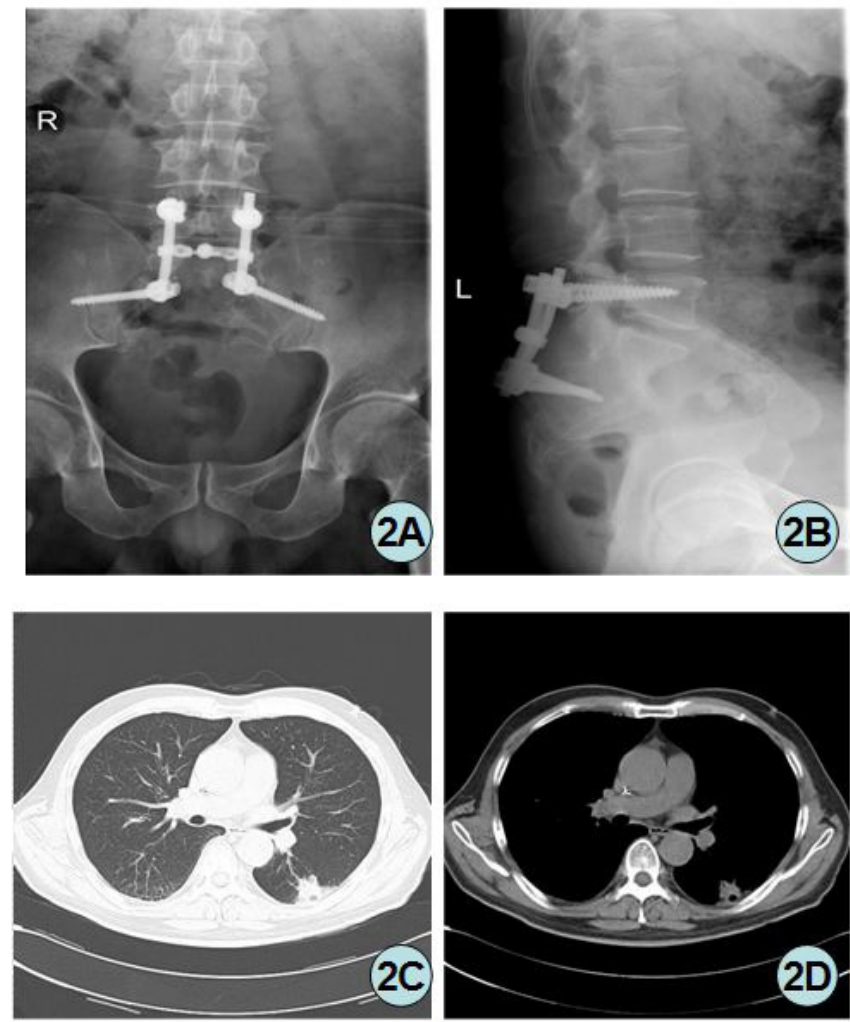

Figure 2

Figure 2A,2B: Anteroposterior and lateral radiographs show the changes after S2 metastatic tumor excision and lumbosacral spine stabilization applied rods and screws fixation. Figure 2C,2D: CT scan shows the nodule located in the left lower lobe slightly larger than before. especially in the right side on $34^{\text {th }}$ day since he received erlotinib. Because it appeared that the patient could not tolerate a bronchoscopy and transbronchial lung biopsy, eILD was impressed without a pathologic support. Immediate withdrawal of erlotinib and administration of methylprednisolone $2 \mathrm{mg} /$ $\mathrm{kg} / \mathrm{d}$ was prescribed for 5 days with dosage gradually reduction, along with supplemental oxygen, nebulisers and antibiotics. General conditions of the patient were deteriorated in the following days, as well as dyspnea and progressive respiratory failure developed. The patient died 3 weeks later (55 days after commencing erlotinib). No postmortem autopsy was obtained, due to his family request.
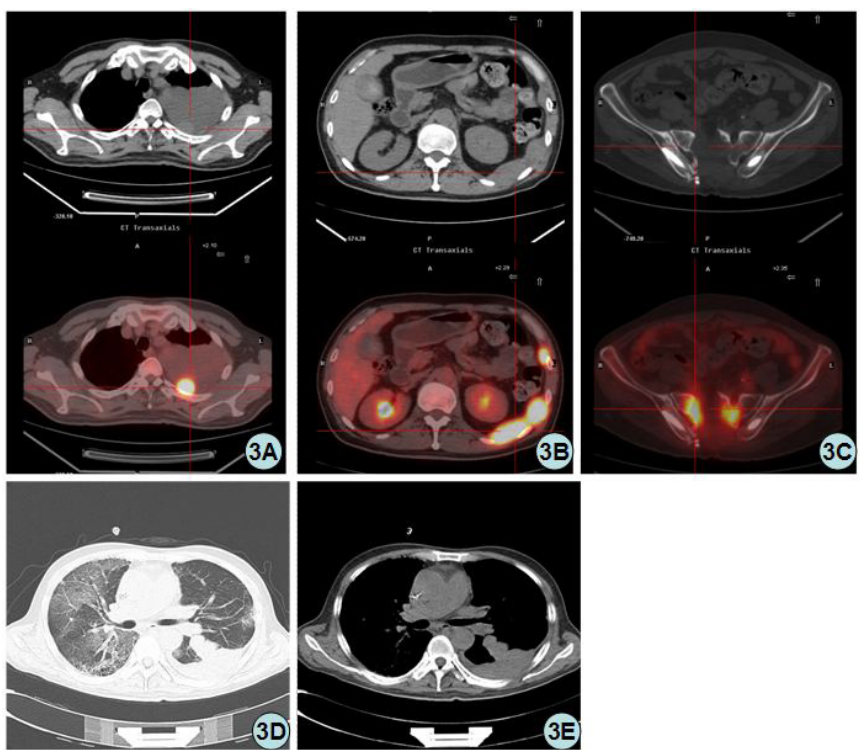

Figure 3

Figure 3A,3B: PET-CT shows multiple soft-tissue density nodules in the left pleura with intense hypermetabolism and massive pleural effusion, a finding highly suspicious for extensive metastasis in the left pleura. Figure 3C: PET-CT shows intense hypermetabolism in the periphery of metastatic tumor excision area in S2, which suggests local recurrence of metastatic tumor in S2. Figure 3D,3E: Chest CT reveals extensive bilateral ground-glass opacities especially in the right side after 34 days of erlotinib therapy. A persistent moderate left-sided pleural effusion is also noted.

\section{Discussion}

The most common adverse events related to erlotinib treatment are skin rash and diarrhea, while the most serious adverse event reported should be eILD. The incidence of eILD was about $0.2-6.5 \%$ in different studies, of which, the highest one lied in Asian, especially in the Japanese [5-11]. While the incidence of all-grade eILD in non-Asian countries was $0.9 \%$. Similarly, treatment with erlotinib significantly increased the risk of developing high-grade ILD. Results indicated that the OR of eILD did not significantly associate with treatment duration of EGFR-TKIs [12]. The reason for this geographic difference is unclear, different genetic background in different population maybe partially explain the above difference [13]. The allele frequency of ABCG2C>A in Japanese $(\sim 30 \%)$ is higher than that in whites $(\sim 10 \%)$. This difference might be related to higher incidence of eILD events in Japanese patients $(\sim 5 \%)$ than in patients in the United States $(\sim 1 \%)$ [14].

eILD started from 5 days to $>9$ months (median 47 days) after initiating erlotinib therapy [15], and developed within 28 
days after the start of treatment in most patients [16]. As to the patient in our report, the eILD was confirmed by HRCT and clinical symptoms 34 days after he received erlotinib, which in accordance with the report mentioned in the literature above. Of note, eILD was fatal in at least one third of these cases [17], the mortality is even higher near to $50 \%$ [18].

The exact mechanism of eILD has not been fully elucidated. A Japanese postmarketing surveillance study of erlotinib yielded four risk factors for the development or exacerbation of eILD: smoking history, Eastern Cooperative Oncology Group (ECOG) performance status (PS) 2 to 4 , concurrent or previous ILD, and concurrent or previous lung infection [8]. The amount of residual normal lung $(\leq 50 \%)$ was identified as risk factors for the development of eILD [16]. Relation between high plasma erlotinib concentrations and the development of eILD has been suggested [19,20]. The ABCB1 1236TT2677TT-3435TT genotype was associated with higher plasma concentration and the risk of developing higher toxicity in patients treated with erlotinib [21]. Increased KL-6 serum level, which is a marker of type II pneumocyte injury, has been described in eILD [22]. EGFR inhibition by erlotinib may thus impair EGF-induced repair by pneumocytes [23]. Modulation of the metabolic pathway of erlotinib due to CYP1A and CYP3A may influence its toxicity [24]. The ATP-binding cassette subfamily G member 2 (ABCG2) 421C >A polymorphism can influence the apparent clearance of erlotinib in patients with NSCLC and may thus be the underlying cause of severe eILD [20]. There was a tendency for eILD to develop more readily when $\mathrm{C} 3$ levels were higher than the median and when $\mathrm{C} 4 \mathrm{~A} /$ $\mathrm{C} 4 \mathrm{~B}$ and APOA1 levels were lower than the median [11]. We could not detect erlotinib plasma concentration, KL-6, C3, C4A/C4B, APOA1 level, and ABCG2 polymorphism of the patient in our report, so we can not clarify the exact mechanism of eILD for him. The patient was a heavy smoker, which may have increased his risk of developing eILD.

Radiologic changes of eILD are nonspecific and seem to be similar to those of other drug-associated lung injury. The CT features of target agent-related ILDs can be classified into six categories: (1) diffuse alveolar damage or acute interstitial pneumonia, (2) bronchiolitis obliterans, (3) cryptogenic organizing pneumonia (COP) or COP-like pattern, (d) hypersensitivity pneumonitis, (5) interstitial pneumonia of either nonspecific interstitial pneumonia or usual interstitial pneumonia pattern, and (6) progressive disease of underlying ILD [25,26]. Erlotinib can cause Radiation Recall Pneumonitis after Palliative Definitive Radiotherapy [27]. The severity of eILD was classified as mild (abnormalities in $<5 \%$ of bilateral lobes), moderate (abnormalities in $5 \%-20 \%$ of bilateral lower lobes), or severe (abnormalities in $>20 \%$ of bilateral lower lobes) [16]. It is self-evident that the patient in our report belongs to the severe one.

eILD remains a poorly understood disease entity. The sparse data available comes mainly from case reports, retrospective trial analyses and postmarketing safety information, predominantly from Japanese patients.

Diagnosis of eILD is often difficult and is based on clinical, radiologic and, where available, pathologic observations. Ob- taining histological samples is not always possible due to the poor health of the patients, and so the diagnosis is often one of exclusion. High suspicion is necessary in those patients under treatment with erlotinib who develop respiratory symptoms like dyspnea, cough or fever $[3,28,29]$. Diagnostic imaging is important primarily to provide an understanding of the baseline pulmonary status and radiographic pattern after onset, determining the severity and extent of the lesions, and assessing the treatment response [16]. In our report, the patient didn't have any hints of congestive heart failure, lung infection or lymphangitic carcinomatosis. His radiologic signs of ILD were apparent after 34 days of erlotinib treatment. We just thought that his respiratory symptoms, such as cough and dyspnea, were the result of pleural effusion. We should give him CT detection earlier, and find clues of ILD earlier.

There have been no randomized controlled or prospective trials to guide the management of eILD. Treatment of eILD is largely supportive, including supplemental oxygen and mechanical ventilation. Immediate discontinuation of the offending drug is recommended. Dose-reduction plays a limited role in preventing recurrence [30]. Systemic corticosteroids are usually prescribed when infection is ruled out, although no controlled trials have been conducted to evaluate their benefit $[31,32]$. Although resolution has been reported, many patients die of progressive respiratory failure [25]. As to the patient in our report, corticosteroids was ineffective, which was the same as the reported data [33].

Although several studies to date have reported on the predictive factors for eILD, it is currently difficult to prevent eILD. In conclusion, the incidence of eILD seems to be low, more cases could be expected with increasing number of patients receiving erlotinib. Clinicians should be aware of clinical and radiographic presentation of eILD. Erlotinib should be discontinued if the patient develops cough and dyspnea. Corticosteroids should be considered after the exclusion of infection, cardiogenic pulmonary edema, diffuse alveolar hemorrhage and lymphangitic carcinomatosis. Further research is needed to identify the risk factors and pathophysiology. This will guide patient selection for erlotinib therapy and help develop more effective treatment strategies [34].

\section{Authors' Contribution}

Ling-chuan Guo and Xi-ming Wang contributed equally to this work as co-first authors

\section{Disclosure}

The authors do not have any conflict of interest.

\section{References}

1) Liang W, Wu X, Fang W, Zhao Y, Yang Y, et al. (2014) Network meta-analysis of erlotinib, gefitinib, afatinib and icotinib in patients with advanced non-small-cell lung cancer harboring EGFR mutations. PLoS One 9:e85245.

2) Mok T, Wu YL, Au JS, Zhou C, Zhang L, et al. (2010) Efficacy and safety of erlotinib in 1242 East/South-East Asian patients with advanced non-small cell lung cancer. J Thorac Oncol 5:1609-1615.

3) Reck M, Mok T, Wolf J, Heigener D, Wu YL (2011) Reviewing the safety of erlotinib in non-small cell lung cancer. Expert Opin Drug Saf 10:147-157. 
4) Tammaro KA, Baldwin PD, Lundberg AS (2005) Interstitial lung disease following erlotinib (Tarceva) in a patient who previously tolerated gefitinib (Iressa). J Oncol Pharm Pract 11:127-130.

5) Kubota K, Nishiwaki Y, Tamura T, Nakagawa K, Matsui K, et al. (2008) Efficacy and safety of erlotinib monotherapy for Japanese patients with advanced non-small cell lung cancer: a phase II study. J Thorac Oncol 3:1439-1445.

6) Takahashi T, Yamamoto N, Nukiwa T, Mori K, Tsuboi M, et al. (2010) Phase II study of erlotinib in Japanese patients with advanced non-small cell lung cancer. Anticancer Res 30:557-563.

7) Nasrallah H, Bar-Sela G, Haim N (2012) Fatal interstitial lung disease associated with gemcitabine and erlotinib therapy for lung cancer. Med Oncol 29:212-214.

8) Nakagawa K, Kudoh S, Ohe Y, Johkoh T, Ando M, et al. (2012) Postmarketing surveillance study of erlotinib in Japanese patients with non-small-cell lung cancer (NSCLC): an interim analysis of 3488 patients (POLARSTAR). J Thorac Oncol 7:1296-1303.

9) Ren S, Li Y, Li W, Zhao Z, Jin C, et al. (2012) Fatal asymmetric interstitial lung disease after erlotinib for lung cancer. Respiration 84:431-435.

10) D’Alessandris QG, Pallini R (2013) Response to: "rare serious complications of erlotinib therapy”. Acta Neurochir (Wien) 155:745.

11) Atagi $S$, Katakami N, Yoshioka $H$, Fukuoka M, Kudoh $S$, et al. (2013) Nested case control study of proteomic biomarkers for interstitial lung disease in Japanese patients with non-small-cell lung cancer treated with erlotinib: a multicenter phase IV study (JO21661). Clin Lung Cancer 14:407-417.

12) Qi WX, Sun YJ, Shen Z, Yao Y (2015) Risk of interstitial lung disease associated with EGFR-TKIs in advanced non-small-cell lung cancer: a meta-analysis of 24 phase III clinical trials. J Chemother 27:40-51.

13) Shi L, Tang J, Tong L, Liu Z (2014) Risk of interstitial lung disease with gefitinib and erlotinib in advanced non-small cell lung cancer: a systematic review and meta-analysis of clinical trials. Lung Cancer 83:231-239.

14) Fujita K, Hirose T, Kusumoto $S$, Sugiyama T, Shirai T, et al. (2014) High exposure to erlotinib and severe drug-induced interstitial lung disease in patients with non-small-cell lung cancer. Lung Cancer 86:113-114.

15) Cohen MH, Johnson JR, Chen YF, Sridhara R, Pazdur R (2005) FDA drug approval summary: erlotinib (Tarceva) tablets. Oncologist 10:461-466.

16) Johkoh T, Sakai F, Kusumoto $M$, Arakawa H, Harada R, et al. (2014) Association Between Baseline Pulmonary Status and Interstitial Lung Disease in Patients With Non-Small Cell Lung Cancer Treated With Erlotinib-A Cohort Study. Clin Lung Cancer 15:448454.

17) Kunkel J, Loddenkemper C, Grozdanovic Z, Schulze K, Schmittel A, et al. (2011) Interstitial lung disease under erlotinib plus gemcitabine for pancreatic carcinoma: a therapeutic dilemma. Pancreas 40:170-171.

18) Yoneda KY, Hardin KA, Gandara DR, Shelton DK (2006) Interstitial lung disease associated with epidermal growth factor receptor tyrosine kinase inhibitor therapy in non-small-cell lung carcinoma. Clin Lung Cancer 8:S31-35.

19) Tsubata $Y$, Hamada A, Sutani A, Isobe T (2012) Erlotinib-induced acute interstitial lung disease associated with extreme elevation of the plasma concentration in an elderly non-small-cell lung cancer patient. J Cancer Res Ther 8:154-156.

20) Fukudo M, Ikemi Y, Togashi Y, Masago K, Kim YH, et al. (2013) Population pharmacokinetics/pharmacodynamics of erlotinib and pharmacogenomic analysis of plasma and cerebrospinal fluid drug concentrations in Japanese patients with non-small cell lung cancer. Clin Pharmacokinet 52:593-609.

21) Hamada A, Sasaki J, Saeki S, Iwamoto N, Inaba M, et al. (2012) Association of $\mathrm{ABCB} 1$ polymorphisms with erlotinib pharmacokinetics and toxicity in Japanese patients with non-small-cell lung cancer. Pharmacogenomics 13:615-624.

22) Vahid B, Esmaili A (2007) Erlotinib-associated acute pneumonitis: report of two cases. Can Respir J 14:167-170.

23) Higenbottam T, Kuwano K, Nemery B, Fujita Y (2004) Understanding the mechanisms of drug-associated interstitial lung disease. Br J Cancer 91:S31-37.

24) Li X, Kamenecka TM, Cameron MD (2010) Cytochrome P450mediated bioactivation of the epidermal growth factor receptor inhibitor erlotinib to a reactive electrophile. Drug Metab Dispos 38:1238-1245.

25) Lind JS, Smit EF, Grünberg K, Senan S, Lagerwaard FJ (2008) Fatal interstitial lung disease after erlotinib for non-small cell lung cancer. J Thorac Oncol 3:1050-1053.

26) Min JH, Lee HY, Lim H, Ahn MJ, Park K, et al. (2011) Druginduced interstitial lung disease in tyrosine kinase inhibitor therapy for non-small cell lung cancer: a review on current insight. Cancer Chemother Pharmacol 68:1099-1109.

27) Onal C, Abali H, Koc Z, Kara S (2012) Radiation recall pneumonitis caused by erlotinib after palliative definitive radiotherapy. Onkologie 35:191-194.

28) Taj A, Kanjwal S, Hammersley JR (2011) New-onset acute interstitial lung disease after treatment with erlotinib in a patient with metastatic squamous cell carcinoma of the lung. Am J Ther 18:e19-21.

29) Del Castillo Y, Espinosa P, Bodí F, Alcega R, Muñoz E, et al. (2010) Interstitial lung disease associated to erlotinib treatment: a case report. Cases J 3:59.

30) Togashi Y, Masago K, Hamatani Y, Sakamori Y, Nagai H, et al. (2012) Successful erlotinib rechallenge for leptomeningeal metastases of lung adenocarcinoma after erlotinib-induced interstitial lung disease: a case report and review of the literature. Lung Cancer 77:464468.

31) Boeck S, Hausmann A, Reibke R, Schulz C, Heinemann V (2007) Severe lung and skin toxicity during treatment with gemcitabine and erlotinib for metastatic pancreatic cancer. Anticancer Drugs 18:11091111.

32) Lai YC, Lin PC, Lai JI, Hsu SY, Kuo LC, et al. (2011) Successful treatment of erlotinib-induced acute hepatitis and acute interstitial pneumonitis with high-dose corticosteroid: a case report and literature review. Int J Clin Pharmacol Ther 49:461-466.

33) Makris D, Scherpereel A, Copin MC, Colin G, Brun L, et al. (2007) Fatal interstitial lung disease associated with oral erlotinib therapy for lung cancer. BMC Cancer 7:150.

34) ter Heine R, van den Bosch RT, Schaefer-Prokop CM, Lankheet NA, Beijnen JH, et al. (2012) Fatal interstitial lung disease associated with high erlotinib and metabolite levels. A case report and a review of the literature. Lung Cancer 75:391-397.

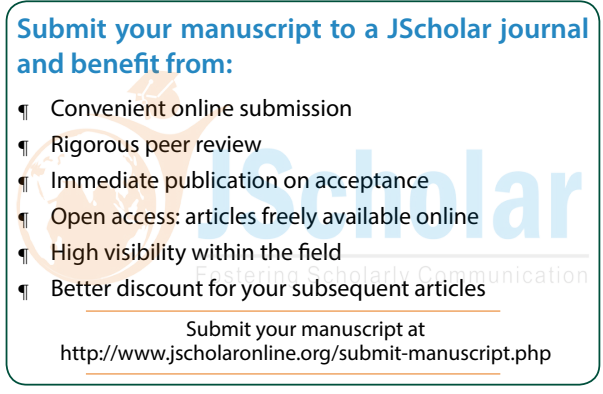

